\title{
"Backward Differential Flow" May Not Converge to a Global Minimizer of Polynomials
}

\author{
Orhan Arıkan' ${ }^{1}$. Regina S. Burachik ${ }^{2}$. \\ C. Yalçın Kaya ${ }^{2}$
}

Received: 8 November 2014 / Accepted: 11 March 2015 / Published online: 20 March 2015

(C) Springer Science+Business Media New York 2015

\begin{abstract}
We provide a simple counter-example to prove and illustrate that the backward differential flow approach, proposed by Zhu, Zhao and Liu for finding a global minimizer of coercive even-degree polynomials, can converge to a local minimizer rather than a global minimizer. We provide additional counter-examples to stress that convergence to a local minimum via the backward differential flow method is not a rare occurence.
\end{abstract}

Keywords Polynomial optimization - Global optimization · Trajectory methods

\section{Introduction}

In their recent article, Zhu et al. [1] provide a method for finding a solution to global minimization of multivariate polynomials of even degree. In this note, we exemplify, and thus prove, that their method does not necessarily yield a global minimizer.

C. Yalçın Kaya

yalcin.kaya@unisa.edu.au

Orhan Arıkan

oarikan@ee.bilkent.edu.tr

Regina S. Burachik

regina.burachik@unisa.edu.au

1 Electrical and Electronics Engineering Department, Bilkent University, Bilkent 06800, Ankara, Turkey

2 School of IT and Mathematical Sciences, University of South Australia, Mawson Lakes, SA 5095, Australia 


\section{Preliminaries}

For simplicity, we focus on the special case of monic quartic univariate polynomials $f: \mathbb{R} \rightarrow \mathbb{R}$ such that

$$
f(x)=x^{4}+a_{3} x^{3}+a_{2} x^{2}+a_{1} x+a_{0},
$$

where $a_{0}, a_{1}, a_{2}$ and $a_{3}$ are real numbers. What Zhu et al. propose in [1] can be translated into this setting as one of solving the following initial value problem. With $x: \mathbb{R} \rightarrow \mathbb{R}$ as the dependent variable and $t$ as the independent variable,

$$
\dot{x}(t)=-\frac{x(t)}{f^{\prime \prime}(x(t))+t}, \quad 0 \leq t \leq t_{0}, \quad x\left(t_{0}\right)=x_{0},
$$

where $\dot{x}=\mathrm{d} x / \mathrm{d} t$, such that

$$
f^{\prime}\left(x_{0}\right)+t_{0} x_{0}=0
$$

and

$$
f^{\prime \prime}(x)+t_{0}>0, \quad \text { for all } x \in \mathbb{R} .
$$

Theorem 4.1 in [1], which is the main result for the so-called backward differential flow method, can then be rephrased as follows.

"If $x(t)$ solves (1) and $f^{\prime \prime}(x(t))+t>0$ for all $\left.\left.t \in\right] 0, t_{0}\right]$, then $x(0)$ is a global minimizer of $f(x)$."

We note that, because $f$ is a monic quartic polynomial, and so is coercive, a large enough positive $t_{0}$ can always be found so that Condition (3) is satisfied. Zhu et al. provide an estimate of $t_{0}$ by restricting the domain of $f$ to a closed ball (in the univariate case, $-a \leq x \leq a)$, in which a global minimizer is contained. In the quartic univariate case, one can even find the smallest $t_{0}$ satisfying (3) easily (as illustrated in the counter-example below). Therefore, an estimate for $t_{0}$ as proposed in [1] is not needed. Then, by (3), there exists a unique solution $x_{0}$ to (2). Finally, the initial value problem (1) is solved from $x\left(t_{0}\right)=x_{0}$ backward in $t$, with the resulting solution referred to as backward differential flow by Zhu et al., to obtain $x(0)$. The point $x(0)$ is claimed in [1] to be a global minimizer. We will prove, via a counter-example, that $x(0)$ is not necessarily a global minimizer.

Before providing a counter-example to Theorem 4.1 of [1], we will make some remarks in order to view the problem from a slightly different point.

Remark 2.1 Define

$$
\varphi(x, t):=f(x)+\frac{t}{2} x^{2} .
$$

Then, $\varphi(x, t)$ can be viewed as a quadratic regularization of $f(x)$, with regularization parameter $t>0$. Note that $\varphi_{x}(x, t)=f^{\prime}(x)+t x$ and $\varphi_{x x}(x, t)=f^{\prime \prime}(x)+t$, where the subscripts $x$ and $x x$ stand for $\partial / \partial x$ and $\partial^{2} / \partial x^{2}$, respectively. Therefore, (2)-(3) above can be rewritten as 


$$
\varphi_{x}\left(x_{0}, t_{0}\right)=0
$$

and

$$
\varphi_{x x}\left(x, t_{0}\right)>0, \quad \text { for all } x \in \mathbb{R} \text {. }
$$

We now recall a well-known fact regarding maximal extension of solutions of ODEs.

Remark 2.2 Assume that $f: \mathbb{R} \rightarrow \mathbb{R}$ is twice continuously differentiable everywhere. Let $t_{0} \in \mathbb{R}$ and $x_{0} \in \mathbb{R}$ such that

$$
f^{\prime}\left(x_{0}\right)+t_{0} x_{0}=0 \text { and } f^{\prime \prime}\left(x_{0}\right)+t_{0}>0 .
$$

The following hold.

(a) There exists $r>0$ such that there is a unique solution $x(\cdot)$ of (1) in $] t_{0}-r, t_{0}+r[$.

(b) There exists a maximal interval to the left of $t_{0}$, say $\left.] m_{0}, t_{0}\right]$, such that there exists a solution of (1) in $\left.] m_{0}, t_{0}\right]$.

(c) Either $m_{0}=-\infty$, or $m_{0} \in \mathbb{R}$ and $f^{\prime \prime}\left(x\left(m_{0}\right)\right)+m_{0}=0$.

Part (a) follows from the classical Picard-Lindelöf existence and uniqueness theorem (see [2]), because the right-hand side of the ODE in (1) is Lipschitz continuous in $x$ and continuous in $t$ in a neighborhood of $t_{0}$. Part (b) is the classical result on maximal extension of solutions of ODEs. The option $m_{0}=-\infty$ of part (c) corresponds to the case in which the right-hand side remains Lipschitz continuous in $x$ for all $t<t_{0}$. The remaining option happens when the denominator

$$
q(t):=f^{\prime \prime}(x(t))+t
$$

vanishes at $t=m_{0}$.

In the following simple lemma, we state a straightforward reformulation of the initial value problem in (1).

Lemma 2.1 Assume that $f: \mathbb{R} \rightarrow \mathbb{R}$ is twice continuously differentiable everywhere. Let $t_{0} \in \mathbb{R}$ and $x_{0} \in \mathbb{R}$ be chosen as in (4). Let $x(\cdot)$ be the maximally extended solution of (1), and $\left.] m_{0}, t_{0}\right]$ the corresponding maximal interval. Then, we have that

$$
\begin{aligned}
\varphi_{x}(x(t), t) & =f^{\prime}(x(t))+t x(t)=0, \quad \varphi_{x x}(x(t), t) \\
& =f^{\prime \prime}(x(t))+t>0, \forall t \in\left[m_{0}, t_{0}\right] .
\end{aligned}
$$

Proof Solvability of (1) over $\left.] m_{0}, t_{0}\right]$ implies that the right-hand side of the ODE is continuous on $\left.] m_{0}, t_{0}\right]$. In other words, the denominator of the right-hand side of the ODE is not zero and so it does not change sign on $\left.] m_{0}, t_{0}\right]$. Since $\varphi_{x x}\left(x\left(t_{0}\right), t_{0}\right)>0$ and the solution exists in $\left.] m_{0}, t_{0}\right]$, we must have

$$
\varphi_{x x}(x(t), t)=f^{\prime \prime}(x(t))+t>0,
$$


for all $\left.t \in] m_{0}, t_{0}\right]$. Then, for all $\left.\left.t \in\right] m_{0}, t_{0}\right]$, we can rewrite the ODE in (1) as

$$
\dot{x}(t)\left(f^{\prime \prime}(x(t))+t\right)+x(t)=0,
$$

which can be rewritten in terms of $\varphi$ as

$$
\frac{\mathrm{d}}{\mathrm{d} t} \varphi_{x}(x(t), t)=0
$$

By (4), we also have

$$
\varphi_{x}\left(x\left(t_{0}\right), t_{0}\right)=f^{\prime}\left(x\left(t_{0}\right)\right)+x\left(t_{0}\right) t_{0}=0 .
$$

Equalities (7) and (8) imply that

$$
\varphi_{x}(x(t), t)=f^{\prime}(x(t))+x(t) t=0,
$$

for all $\left.t \in] m_{0}, t_{0}\right]$. Equality (9) holds at $t=m_{0}$ by continuity of $f^{\prime}$ and $x(\cdot)$.

Next lemma shows that if we start with a negative initial value at $t_{0}$, then the solution of the initial value problem (1) remains negative over its maximal domain of definition.

Lemma 2.2 Let $f: \mathbb{R} \rightarrow \mathbb{R}$ be twice continuously differentiable everywhere. Let $t_{0} \in \mathbb{R}$ and $x_{0} \in \mathbb{R}$ be chosen as in (4). Consider the initial value problem (1). Let $x(\cdot)$ be the maximally extended solution of (1), and $\left.] m_{0}, t_{0}\right]$ the corresponding (finite or infinite) maximal interval of definition of $x(\cdot)$. If $x_{0}<0$, then $x(t)<0$ for all $\left.t \in] m_{0}, t_{0}\right]$. If $m_{0} \in \mathbb{R}$, then $x\left(m_{0}\right)<0$.

Proof Suppose that for some $\left.t \in] m_{0}, t_{0}\right]$, we have $x(t) \geq 0$. Consider the set $S:=$ $\left.\left.\{t \in] m_{0}, t_{0}\right]: x(t) \geq 0\right\}$. This set is non-empty and bounded above by $t_{0}$. Let

$$
t_{1}:=\sup S
$$

Note that $t_{1} \in S$ and $t_{1}<t_{0}$. We claim that $x\left(t_{1}\right) \geq 0$. Indeed, if $x\left(t_{1}\right)<0$, then for some $r>0$, we have

$$
x(t)<0, \text { for all } t \in] t_{1}-r, t_{1}+r[.
$$

By definition of $t_{1}$ as a supremum of $S$, there exists $t \in S$ such that $\left.\left.t \in\right] t_{1}-r, t_{1}\right]$, which means that $x(t) \geq 0$, contradicting (10). Hence, $x\left(t_{1}\right) \geq 0$ and by definition of $t_{1}$, we have

$$
\left.x(t)<0, \text { for all } t \in] t_{1}, t_{0}\right] .
$$

Using (11) and Lemma 2.1 in the ODE in (1), we conclude that

$$
\left.\dot{x}(t)>0 \text {, for all } t \in] t_{1}, t_{0}\right] \text {. }
$$


By the mean value theorem, there exists $\left.s \in] t_{1}, t_{0}\right]$ such that

$$
x\left(t_{1}\right)=x\left(t_{0}\right)+\dot{x}(s)\left(t_{1}-t_{0}\right)<x_{0},
$$

where we used (12). The above expression implies that

$$
\left.\left.x\left(t_{1}\right)<x_{0}<0, \text { for all } t \in\right] t_{1}, t_{0}\right]
$$

which is a contradiction. Hence, $x(t)<0$, for all $\left.t \in] m_{0}, t_{0}\right]$. To prove the last assertion of the lemma, assume on the contrary that $x\left(m_{0}\right) \geq 0$. Since $x(t)<0$, for all $\left.t \in] m_{0}, t_{0}\right]$, use again Lemma 2.1 in the ODE in (1), to obtain (12) with $m_{0}$ in the place of $t_{1}$. Using the mean value theorem again, we get

$$
0 \leq x\left(m_{0}\right)=x\left(t_{0}\right)+\dot{x}(s)\left(m_{0}-t_{0}\right)<x_{0}<0,
$$

for some $\left.s \in] m_{0}, t_{0}\right]$. The above expression entails a contradiction, which implies that $x\left(m_{0}\right)<0$.

Lemma 2.3 Let $f: \mathbb{R} \rightarrow \mathbb{R}$ be twice continuously differentiable everywhere. Let $t_{0} \in \mathbb{R}$ and $x_{0} \in \mathbb{R}$ be chosen as in (4). Consider the initial value problem (1) with $x_{0}<0$. Assume that the system, with the unknown $(x, t) \in \mathbb{R}^{2}$, given by

$$
f^{\prime}(x)+t x=0, \quad f^{\prime \prime}(x)+t=0
$$

has a unique real solution $(\bar{x}, \bar{t})$ with $\bar{x}>0$ and $\bar{t}>0$. Then, the solution of (1) can be infinitely extended to the left; in other words, $m_{0}=-\infty$, and so $x(t)<0$, for all $t \leq t_{0}$.

Proof Indeed, assume that, on the contrary, $m_{0} \in \mathbb{R}$. By Remark 2.2(c), this can only happen if the right-hand side of (1) becomes discontinuous at $t=m_{0}$. This implies that

$$
f^{\prime \prime}\left(x\left(m_{0}\right)\right)+m_{0}=0 .
$$

By Lemma 2.1, we have

$$
f^{\prime}(x(t))+t x(t)=0
$$

for all $t \in\left[m_{0}, t_{0}\right]$. This fact combined with (4) implies that

$$
f^{\prime}\left(x\left(m_{0}\right)\right)+m_{0} x\left(m_{0}\right)=0 .
$$

By Lemma 2.2, we have that $x\left(m_{0}\right)<0$. Equations (15) and (16) imply that there is a pair $(x, t)=\left(x\left(m_{0}\right), m_{0}\right)$ which solves system (14), with $x<0$. Since system (14) has a unique solution $(\bar{x}, \bar{t})$ with $\bar{x}>0$, we arrive at a contradiction. Hence, we must have $m_{0}=-\infty$. It follows by Lemma 2.2 that $x(t)<0$, for all $t \leq t_{0}$. 


\section{Counter-Example}

\section{Proposition 3.1 Consider}

$$
f(x)=x^{4}-8 x^{3}-18 x^{2}+56 x .
$$

Suppose that $x(t)$ solves (1). Then, one has that $f^{\prime \prime}(x(t))+t>0$ for all $\left.\left.t \in\right] 0, t_{0}\right]$, but that $x(0)$ is not a global minimizer of $f(x)$.

Proof We will first show that this quartic polynomial function $f(x)$ verifies the hypotheses of Lemma 2.3. Then, we will conclude that there exists $t_{0}$ such that the denominator $q(t)$, defined in (5), is positive for all $\left.t \in \models \infty, t_{0}\right]$. Hence, $f(x)$ satisfies the assumptions of Theorem 4.1 in [1].

Note that $f(x)$ has local minima at $x=-2$ and $x=7$ and a local maximum at $x=1$. We also note that $f(-2)=-104, f(7)=-833$ and $f(1)=31$. Therefore, $x=7$ is the global minimizer of $f(x)$.

Let us now compute $t_{0}$ and $x_{0}$. We have

$$
\varphi_{x}\left(x_{0}, t_{0}\right)=4 x_{0}^{3}-24 x_{0}^{2}+\left(t_{0}-36\right) x_{0}+56=0
$$

and

$$
\varphi_{x x}\left(x, t_{0}\right)=12 x^{2}-48 x+t_{0}-36>0, \text { for all } x \in \mathbb{R} .
$$

The minimum of the quadratic function $\varphi_{x x}\left(x, t_{0}\right)$ above occurs at $x=2$. Therefore, one gets $t_{0}>84$, to guarantee that (3) holds. Let $t_{0}=100$. Then we obtain, as the only real solution of (17),

$$
x_{0}=2+((\sqrt{18417} / 9)-15)^{1 / 3}-4 /\left(3((\sqrt{18417} / 9)-15)^{1 / 3}\right)<0
$$

by means of some computer algebra package, e.g., MATLAB. Approximately, $x_{0} \approx$ -0.681220 . The initial value problem (1) becomes

$$
\dot{x}(t)=-\frac{x(t)}{12 x^{2}(t)-48 x(t)+t-36}, \quad 0 \leq t \leq 100, \quad x(100)=x_{0} .
$$

Next, let us show that $f$ verifies the hypotheses of Lemma 2.3. From $\varphi_{x x}(\bar{x}, \bar{t})=0$, which is the second equation of (14), we get

$$
\bar{t}=-12 \bar{x}^{2}+48 \bar{x}+36 \text {. }
$$

Substitution of this expression for $\bar{t}$ into $\varphi_{x}(\bar{x}, \bar{t})=0$, which is the first equation of (14), yields

$$
8 \bar{x}^{3}-24 \bar{x}^{2}-56=0
$$




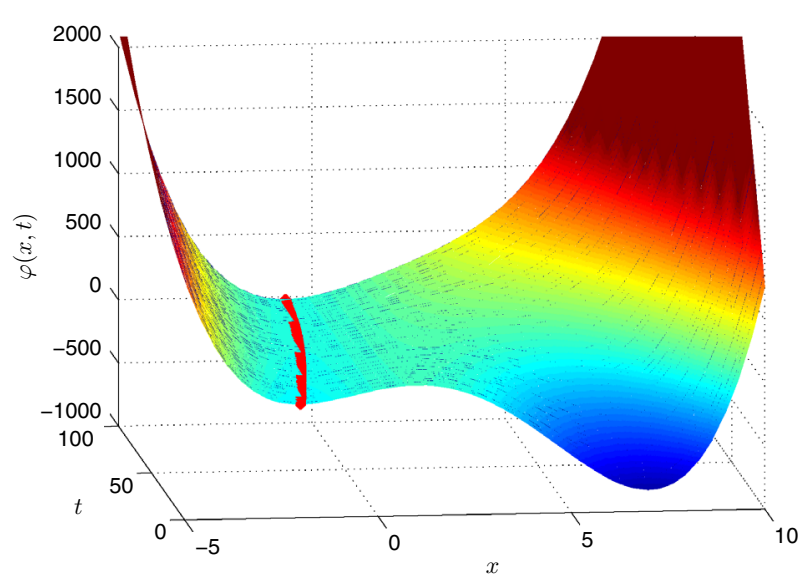

Fig. 1 Backward differential flow for the counter-example, $f(x)=x^{4}-8 x^{3}-18 x^{2}+56 x$

The only real solution of the latter equation is found as

$$
\bar{x}=1+\left(\frac{9+\sqrt{77}}{2}\right)^{1 / 3}-\left(\frac{2}{9+\sqrt{77}}\right)^{1 / 3}>0
$$

by MATLAB. Approximately, $\bar{x} \approx 3.554149$ and, in turn, $\bar{t} \approx 55.01544$.

Therefore, the hypotheses of Lemma 2.3 are satisfied. Note also that the denominator in (5), $q\left(t_{0}\right)=q(100)>0$. Since, by Lemma 2.3, the solution of (18) is well-defined on $-\infty, 100]$, we have that the denominator $q(t)>0$ for all $t \in[0,100]$, satisfying the hypotheses of Theorem 4.1 in [1].

Since $x_{0}<0$ and $q(100)>0$, we have $\dot{x}(100)>0$, and so, by Lemma 2.3 , the unique $x(t)$ which solves (18) is negative for all $t \in[0,100]$. However, $x(0)<0$ is not the global minimizer of $f(x)$.

In Fig. 1, an illustration of the backward differential flow method, as applied to the polynomial in Proposition 3.1, is given. The solution curve of (18) is depicted on a surface plot of the function $\varphi(x, t)$. The curve is generated by solving (18) numerically using the MATLAB function ode113, with RelTol $=1 \mathrm{e}-06$. It can be clearly observed in the figure that $x(0)$ approximates the local minimizer $x=-2$, rather than the global minimizer $x=7$.

\subsection{Other Counter-Examples}

The fact that $x(0)$ is not a global minimizer is not a rare occurence; indeed, it is frequently encountered. In what follows, we provide a few more examples for which $x(0)$ of the backward differential flow is not a global minimizer.

$f(x)=x^{4}-(16 / 3) x^{3}-2 x^{2}+16 x+2$ (global minimizer: $x=4$; local minimizer: $x=-1)$ 
$f(x)=x^{4}+(20 / 3) x^{3}-2 x^{2}-20 x+3$ (global minimizer: $x=-5$; local minimizer: $x=1$ )

\section{Conclusions}

We have demonstrated, via a counter-example, that the backward differential flow approach presented by Zhu et al. [1] does not necessarily yield a global minimizer of a coercive even-degree polynomial. The counter-example will hopefully help/prompt to determine where the proof of Theorem 4.1 in [1] breaks down. This might in turn help find a correct statement for the theorem.

\section{References}

1. Zhu, J., Zhao, S., Liu, G.: Solution to global minimization of polynomials by backward differential flow. J. Optim. Theory Appl. 161, 828-836 (2014)

2. Arnold, V.I.: Ordinary Differential Equations. The MIT Press, Cambridge (1978) 